\title{
Problems in the diagnosis and investigation of aortic stenosis
}

\author{
Andrew Bishop and Peter Wilkinson
}

Ashford Hospital, Ashford, Middlesex TW153AA, UK

Valvular heart disease has changed considerably from the days when the consequences of rheumatic fever dominated the clinical load. Of the four types of aortic stenosis - congenital, rheumatic, calcified bicuspid valve, and senile calcified tricuspid valve the latter two underlie the majority of new presentations at hospital with haemodynamically significant valve lesions. ${ }^{1}$

The disease of calcific aortic stenosis is different from rheumatic aortic stenosis in that the functional obstruction is due to immobility of the calcified cusps rather than commissural fusion. Thus the clinical condition is very slowly progressive, often becoming significant only in old age. In addition the separation of the cusps is directly related to ventricular systolic pressure rather than fixed as with commissural fusion, and thus both valve area and outflow gradient vary with ventricular function from minute to minute. ${ }^{2}$ The central clinical question is therefore where along this continuum of gradually increasing sclerosis and calcification of the valve does aortic stenosis begin?

The pragmatic answer to this question is at the point at which treatment is of potential benefit, and the clinical detection and assessment of aortic stenosis is entirely directed towards isolating those patients who would benefit from intervention. The natural history of symptomatic aortic stenosis is rapidly progressive and relentless, so that in the era before aortic valve surgery a patient with symptoms of angina or syncope was given a prognosis of $3-5$ years, and with dyspnoea up to 2 years. ${ }^{3,4}$ Thus any patient with these symptoms as a result of aortic outflow obstruction would potentially benefit from intervention, and this is borne out in the increasing success of aortic valve replacement in the elderly population. ${ }^{5}$

The subsequent problem is that of the asymptomatic patient with significant aortic stenosis.

Correspondence: P.R. Wilkinson, M.D., F.R.C.P. Received: 7 August 1991
Prospective series describe between $30 \%$ and $40 \%$ of such patients developing symptoms within 2 years of detection, and no deaths without the patient first having developed symptoms. ${ }^{6,7}$ It is clear therefore that further assessment with a view to aortic valve surgery is appropriate for any patient with angina, syncope, or dyspnoea, and physical signs to suggest aortic stenosis.

The physical sign that usually alerts the doctor is a systolic murmur. More detailed attention to other physical signs is held to be helpful in distinguishing a haemodynamically significant lesion, including the pulse width and character, the loudness of the aortic component of the second heart sound, and the character of the apex beat. Further investigations are usually performed specifically to demonstrate aortic valve calcification on chest X-ray, left ventricular hypertrophy on 12 lead electrocardiogram (ECG), and left ventricular hypertrophy, valve thickening and restricted cusp separation on M-mode echocardiography.

In this issue McKillop et al. ${ }^{8}$ present data to show that, in an elderly population, features of the physical examination and routine investigations are neither sensitive nor specific when assessed against a Doppler derived gradient for diagnosing significant aortic stenosis. While this is in keeping with clinical experience, others have reported that a combination of all of these separate features gives a clear guide to the presence of significant disease. This is borne out by a prospective study by Nitta and others ${ }^{9}$ in which a scoring system was employed, giving points for left ventricular hypertrophy on ECG, calcification on the chest X-ray, loudness of the aortic component of the second heart sound, the timing of the peak of the murmur, the speed of rise of carotid pulse, the ejection time, and left ventricular wall thickness on echo. When validated against valve area measurement at cardiac catheterization a score was found that was $93 \%$ sensitive and $96 \%$ specific for a valve area of less than $1 \mathrm{~cm}^{2}$.

A firm assessment of which patients should be referred for interventional management is therefore within the capability of many physicians. 
However, two pieces of data are almost always required before intervention is considered, and there is debate surrounding both. Firstly, a quantitative assessment of the degree of outflow obstruction is made. The valve orifice, and gradient across the valve are the most common measurements, although as described above, the dominant calcific lesion in current practice is characterized by variable gradient and orifice size depending on systolic function. The peak to peak or instantaneous transaortic gradient at cardiac catheterisation is usually taken as the haemodynamic standard for assessing the severity of stenosis. Many studies are now published validating the gradients derived from Doppler echo studies against cardiac catheter data, and it is clear that adequate Doppler recordings can be made in the majority of patients. Doppler seems to be particularly good at differentiating critical from non-critical stenosis and in allowing milder forms to be followed non-invasively. ${ }^{10,11}$ However there are patients in the mid-range of severity on Doppler whose symptoms are uncertain where cardiac catheterization will be needed to define the degree of stenosis with more certainty. ${ }^{12}$

The use of gradient as a measurement of severity of stenosis has been questioned in not only calcific aortic stenosis but also rheumatic stenosis, in that the gradient is dependent on the left ventricular capacity to maintain a fixed cardiac output. The presence of systolic dysfunction, or other valve lesions, notably mitral regurgitation, will lead to underestimation of severity of stenosis by lowering the outflow gradient. Griffith et al..$^{13}$ found difficulty in defining a gradient that specifically and sensitively predicted a valve area compatible with severe aortic stenosis, and concluded that valve area should be measured in all cases. The measurement of valve area, however, using the Gorlin formula, is rendered less accurate by the inherent inaccuracies of pulmonary capillary wedge pressure and cardiac output assessments, and assessment of the method in prosthetic valves where the actual orifice size is known shows that a mean error of $0.36 \mathrm{~cm}^{2}$ is associated with the technique. ${ }^{14}$ Thus

\section{References}

1. Petersen, M.D., Roach, R.M. \& Edwards, J.E. Types of aortic stenosis in surgically removed valves. Arch Pathol Lab Med 1985, 109: 829-832.

2. Selzer, A. Changing aspects of the natural history of valvular aortic stenosis. $N$ Engl J Med 1987, 317: 91-98.

3. Ross, J. Jr \& Braunwald, E. Aortic stenosis. Circulation 1968, 38(Suppl 5): V61-V67.

4. Olesen, K.H. \& Warburg, E. Isolated aortic stenosis - the late prognosis. Acta Med Scand 1957, 160: 437-446.

5. Deleuze, P., Loisance, D.Y., Besnainov, F. et al. Severe aortic stenosis in octogenarians: is operation an acceptable alternative? Ann Thorac Surg 1990, 50: 226-229. a technique for absolutely quantifying aortic气 stenosis is not available, and without detailed 3 information about ventricular function neither $\varnothing$ gradient or valve area are measurements that give a $ᄃ$ definite value above which the valve is judged to be $\overrightarrow{\vec{F}}$ significantly stenosed.

Another potentially useful measurement, partic- $\frac{\bar{O}}{0}$ ularly where clear doppler signals from the stenotic $\frac{\bar{\sigma}}{\bar{c}}$. jet cannot be obtained, is the ejection time and, $\vec{\Phi}$ using its variation from predicted, an estimate of $\varrho$ valve area with $88 \%$ sensitivity, $89 \%$ specificity, and $89 \%$ accuracy can be derived. ${ }^{15}$

With these non-invasive techniques available, the need for invasive investigation of aortic stenosis $\vec{\omega}$ is to a large extent limited to the desire to know the $\frac{}{8}$ coronary anatomy. Conventional wisdom is that $\frac{0}{3}$ the combination of fixed cardiac output, reduced. diastolic duration and ventricular hypertrophy, is. such a potent scenario for the development of 0 angina of effort, that its absence excludes the possibility of significant coronary disease. Many $\vec{\odot}$ cardiologists would therefore reserve cardiac cath- $\odot$ eterization for angina sufferers. A comprehensive 음 retrospective analysis of more than 500 patients who underwent aortic valve replacement showed ${ }_{D}$ that omission of coronary artery bypass grafting $\underset{\mathbb{\Phi}}{\overparen{D}}$ was an independent risk factor for early mortality, while coronary artery disease was a pre-operatice $\Phi$ risk factor for late mortality that was not infle enced by bypass grafting at operation. The eart $\Theta$ mortality was largely confined to a group that haof. high prediction score for coronary artery disease before assessment; ${ }^{16}$ however the score had a predictive value of only $66 \%$, and it therefore remains unclear whether the absence of angina of $\approx$ effort is in itself a sufficiently reliable guide to the $\stackrel{\unrhd}{\stackrel{2}{ }}$ need for cardiac catheterization.

There are thus difficulties in the assessment of the patient with a suspected aortic ejection murmur. While there is little doubt that aortic outflow obstruction causing symptoms is an indication for intervention, establishing the presence of that 3 . obstruction and subsequently quantifying it is less straightforward than it appears.

6. Kelly, T.A., Rothbart, R.M., Cooper, C.M. et al. Comparison of outcome of asymptomatic to symptomatic $\Omega$ patients older than 20 years of age with valvular aortic $N$ stenosis. Am J Cardiol 1988, 61: 123-130.

7. Pellikka, P.A., Bailey, K.R., Nishimura, R.A. et al. The natural history of adults with asymptomatic, severe aortic $\bar{\sigma}$ stenosis. J Am Coll Cardiol 1989, 13: 147A.

8. McKillop, G.M., Stewart, D.A., Burns, J.M.A.\& Ballantyne, D. Doppler echocardiography in elderly patients with ejec- $\mathbb{D}$ tion systolic murmurs. Postgrad Med J 1991, 67: 1059-1061. ? 
9. Nitta, M., Nakamura, T., Hultgren, H.N. et al. Non invasive evaluation of the severity of aortic stenosis in adults. Chest 1987, 91: 682-687.

10. Currie, P.J., Hagler, D.J., Seward, J.B. et al. Instantaneous pressure gradient: a simultaneous doppler and dual catheter correlative study. J Am Coll Cardiol 1986, 7: 800-806.

11. Yeager, M., Yock, P.G. \& Popp, R.L. Comparison of doppler derived pressure gradient to that determined at cardiac catheterisation in adults with aortic valve stenosis: implications for management. Am J Cardiol 1986, 57: 644-648.

12. Galan, A., Zoghbi, W.A. \& Quinones, M.A. Determination of severity of valvular aortic stenosis by doppler echocardiography and relation of findings to clinical outcome and agreement with hemodynamic measurements determined at cardiac catheterisation. Am J Cardiol 1991, 67: 1007-1012.
13. Griffith, M.J., Carey, C., Coltart, D. et al. Inaccuracies in using aortic valve gradients alone to grade severity of aortic stenosis. $\mathrm{Br}$ Heart J 1989, 62: 372-378.

14. Cannon, S.R., Richards, K.L., Crawford, H.N. et al. Inadequacy of the Gorlin formula for predicting prosthetic valve area. Am J Cardiol 1988, 62: 113-116.

15. Zoghbi, W.A., Galan, A. \& Quinones, M.A. Accurate assessment of aortic stenosis severity by doppler echocardiography independent of aortic jet velocity. Am Heart $J$ 1988, 116: 855-863.

16. Lund, O., Nielsen, T.T., Pilegard, H.K. et al. The influence of coronary artery disease and bypass grafting on early and late survival after valve replacement for aortic stenosis. J Thorac Cardiovasc Surg 1990, 100: 327-337. 